\title{
Thermoluminescence response and apparent anomalous fading factor of Durango fluorapatite as a function of the heating rate
}

\author{
G. Kitis ${ }^{1}$, G. S. Polymeris ${ }^{1,3}$, V. Pagonis ${ }^{*}$, , and N. C. Tsirliganis ${ }^{3}$ \\ 1 Aristotle University of Thessaloniki, Nuclear Physics Laboratory, 54124 - Thessaloniki, Greece \\ 2 Physics Department, McDaniel College, Westminster, MD 21158, USA \\ ${ }^{3}$ Cultural and Educational Technology Institute, Archaeometry Laboratory, Tsimiski 58, 67100 - Xanthi, \\ Greece
}

Received 21 April 2006, revised 6 September 2006, accepted 7 September 2006 Published online 20 October 2006

PACS 78.60Kn, 91.60.Mk

The anomalous fading (AF) of the Thermoluminescence (TL) signal has been observed in Durango apatite, and is believed to be caused by electrons transferred to the recombination sites via a tunnelling effect. The AF factor is a property unique for each glow-peak of a glow-curve and shows a remarkable stability, even when extreme experimental conditions are applied to this material. In the present work the AF is studied as a function of the rate at which the material is heated in order to obtain its TL. In this experimental study it is found that the TL response of Durango apatite is drastically increased as the rate of heating increases, contrary to what is expected from standard kinetic models of TL. An important consequence of this result is that, since the AF rate is monitored through the TL signal, the variations of TL as a function of the heating rate will be passed on to the evaluation of the AF rate. The result is an apparent decrease of the AF with an increasing heating rate, although the actual AF effect has no relation with the TL readout process. A possible explanation is proposed for the increase of the TL response and for the apparent decrease of the AF rate with the rate of heating.

(C) 2006 WILEY-VCH Verlag GmbH \& Co. KGaA, Weinheim

\section{Introduction}

Anomalous fading $(\mathrm{AF})$ is the term adopted for the rapid decay at room temperature of the high temperature thermoluminescence (TL) glow-peaks signal, contrary to the expected stability predicted by the basic TL kinetic models $[1,2]$. The AF has been attributed to a temperature independent quantum mechanical tunnelling effect $[3,4]$. However, when the AF is temperature dependent then it has been explained by assuming a thermally assisted tunnelling model [4] or a model which involves localized transitions [5, 6]. Finally, Chen and Hag-Yahya [7] proposed a model, which considers AF as normal fading in disguise.

The ratio of the TL at time $t$ over the initial TL at time $t_{0}$ is known as the remnant TL parameter $r$. Kitis et al. [8] studied the AF rate of Durango apatite as a function of grain size, annealing temperature, pre-dose and irradiation temperature and had found that the $r$ factor is not seriously influenced by all these external parameters with the exception of one case. The AF was found to increase after 1 hour annealing at $1000{ }^{\circ} \mathrm{C}$. Polymeris et al. [9] had shown that both the TL sensitivity and the AF factor of the main TL glow-peak of Durango apatite shows a remarkable stability over many irradiation-storage time$\mathrm{TL}$ readout cycles. Therefore, the AF factor $r$, which is unique for each glow-peak of a glow-curve

\footnotetext{
"Corresponding author: e-mail: vpagonis@mcdaniel.edu, Phone: +001 4108572481
} 
seems to show a remarkable resistance to variation even if extreme external conditions are applied to the material.

In TL studies the AF factor is evaluated by monitoring the amount of TL as a function of storage time after the end of irradiation. However, it is well known that various effects are taking place during TL readout, which are usually called recombination stage effects. Since these effects are temperature dependent, the simplest way to recognize them is to alter the TL readout heating rate, which causes a shift of the glow-curve towards higher temperatures. Two characteristic examples of heating rate effects on the TL of materials are the following: (i) The well known effect of the drastic decrease of the TL response as a function of the heating rate known to occur in some materials [10-12], which has been attributed to the thermal quenching effect. (ii) The superlinear TL dose response of glow-peak 5 in $\mathrm{LiF}: \mathrm{Mg}, \mathrm{Ti}[13]$ and the very strong superlinear $\mathrm{TL}$ dose response of the $110^{\circ} \mathrm{C}$ glow-peak of synthetic quartz [14]. It has been proposed that the latter effect varies strongly with the concentration of the relevant competitor sites [13].

By extrapolating these findings to the AF effect observed in apatite, one might expect a possible apparent change of the AF rate by altering the recombination stage conditions through a variation of the readout heating rate. It must be noted that, in the framework of tunnelling models, the AF effect is also a recombination stage effect, which however, takes place during storage time only.

This paper is concerned with (a) The dependence of the TL response of apatite on the heating rate, which is not predicted by standard kinetic models and (b) The apparent variation of the AF factor in this material with the heating rate.

\section{Material and apparatus}

The study was performed on Durango apatite, a type of apatite which is known to exhibit strong anomalous fading of its TL signal [8]. The sample was initially crushed, and grains with dimensions $2-10 \mu \mathrm{m}$ were selected and deposited on aluminum disks of $1 \mathrm{~cm}^{2}$ area using the Zimmerman method [15]. The grains were annealed at $500{ }^{\circ} \mathrm{C}$ for $1 \mathrm{hr}$.

The measurements were performed using the RISO TL/OSL reader (model Tl/OSL-DA-15) equipped with a $0.085 \mathrm{~Gy} / \mathrm{s}{ }^{90} \mathrm{Sr}-{ }^{90} \mathrm{Y} \beta$-ray source. The TL measurements were performed using a combination of a Pilkington HA-3 heat absorbing and a Corning 7-59 blue filter, using a maximum readout temperature of $500{ }^{\circ} \mathrm{C}$.

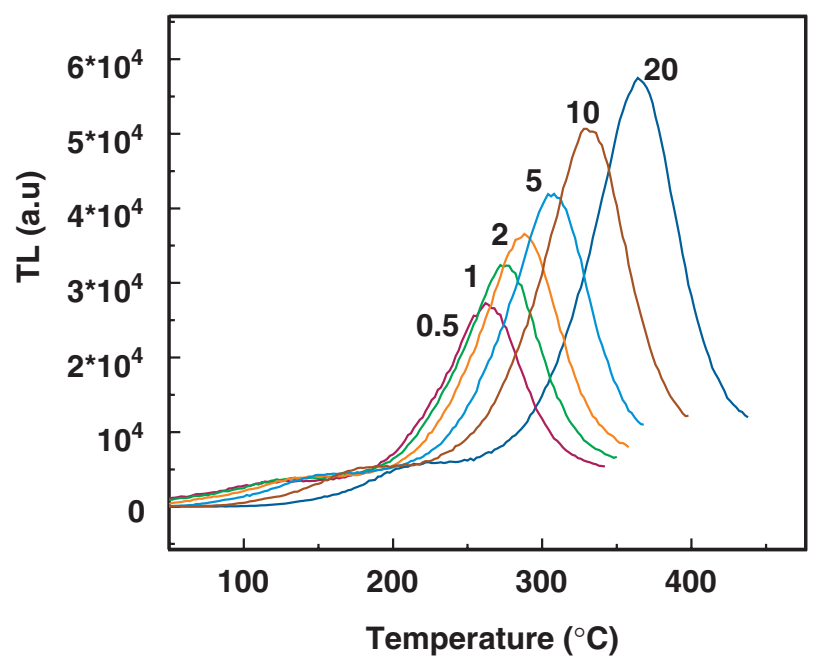

Fig. 1 (online colour at: www.pss-a.com) Glow-curves of Durango apatite as a function of the heating rate, noted on the respective glowcurve, for zero storage time. 


\section{Experimental results}

The measurement protocol used to study the dependence of the AF of TL as a function of the heating rate was as follows.

1. Give a test dose of 15.3 Gy and measure the TL immediately after the end of irradiation, in order to obtain the zero storage time sensitivity. Since the AF is known to be a remarkably stable experimental parameter, this measurement is also used for normalizing the sensitivity of the samples.

2. Give the same test dose and store the sample at dark conditions for a pre-selected period of time $t$ after the end of irradiation.

3. At the end of the storage period measure the TL of the sample with a heating rate $\beta$.

4. Repeat steps 1, 2 and 3 for another storage period of time $t(10,20,40,60,120,240,480 \mathrm{~min})$.

5. Repeat steps $1,2,3$ and 4 for a higher heating rate $\beta$. The values of $\beta$ used were $0.5,1,2,5,10$ and $20^{\circ} \mathrm{C} / \mathrm{s}$.

The glow-curve shapes as a function of the heating rate are shown in Fig. 1. The TL is measured as the integral of the main glow-peak.

It is important to note that one must be very careful in comparing TL glow curves measured with different heating rates. If the integral of the TL glow curve is calculated using a time scale, then one can expect the integral to be constant and independent of the heating rate. If on the other hand, the integral is calculated using a temperature scale, then one must divide the result with the corresponding heating rate before comparing the glow curves. The areas and glow curves being presented in this paper have been divided by the appropriate heating rate, and the glow-curves given in Fig. 1 are directly comparable with each other. Therefore, the increase of the TL as a function of the heating rate is a clear and unambiguous experimental result.

Figure 2 shows the dependence of the integral of the main TL glow-peak of Durango apatite as a function of the heating rate. Each curve corresponds to a different storage time varying from zero minutes for curve (a), up to 480 minutes for curve (h). The result is interesting for two reasons. First, the TL response is significantly increased as a function of the heating rate. This result is not expected from the phenomenological TL kinetic theory, which predicts that the TL response does not depend on the heating rate. Secondly, the dependence of the TL response on the heating rate varies with the storage time used.

The left-hand side Fig. 3 shows the behavior of the maximum temperature of the main glow-peak as a function of the heating rate. The shift towards higher temperatures follows the general trend expected from the phenomenological TL kinetic theory. However, the amount of the shift in maximum temperature is rather high, which indicates that a serious temperature lag effect may exist at higher heating rates $[16,17]$. On the other hand the variation of the Full Width at Half Maximum (FWHM), shown in the right-hand side of Fig. 3, is rather small because the total variation is less than $5{ }^{\circ} \mathrm{C}$, when the heating rate varies from 0.5 to $20^{\circ} \mathrm{C} / \mathrm{s}$. In fact, we may have expected that the variation of the $\mathrm{FWHM}$ would

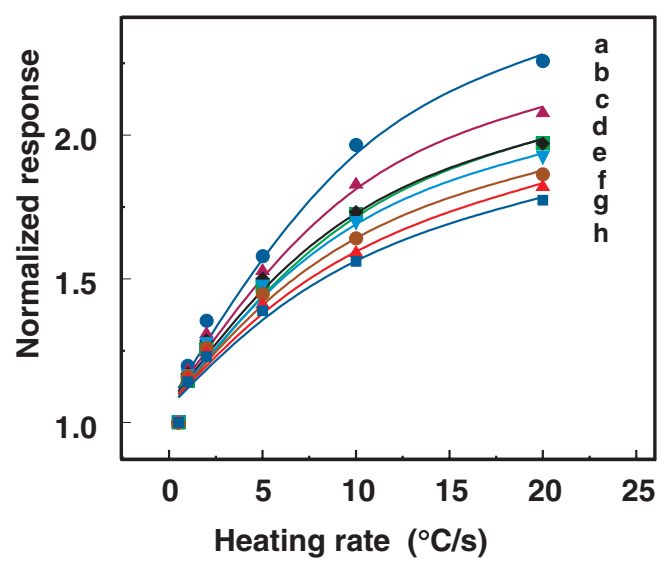

Fig. 2 (online colour at: www.pss-a.com) TL response of the integral of the main glow-peak of Durango apatite, normalized over the lower heating rate, as a function of the heating rate, for various storage times. Curve (a) is for zero storage time, whereas lines $(\mathrm{b}-\mathrm{h})$ correspond to storage times 10, 20, 40, 60, 120, 240 and $480 \mathrm{~min}$, respectively. 

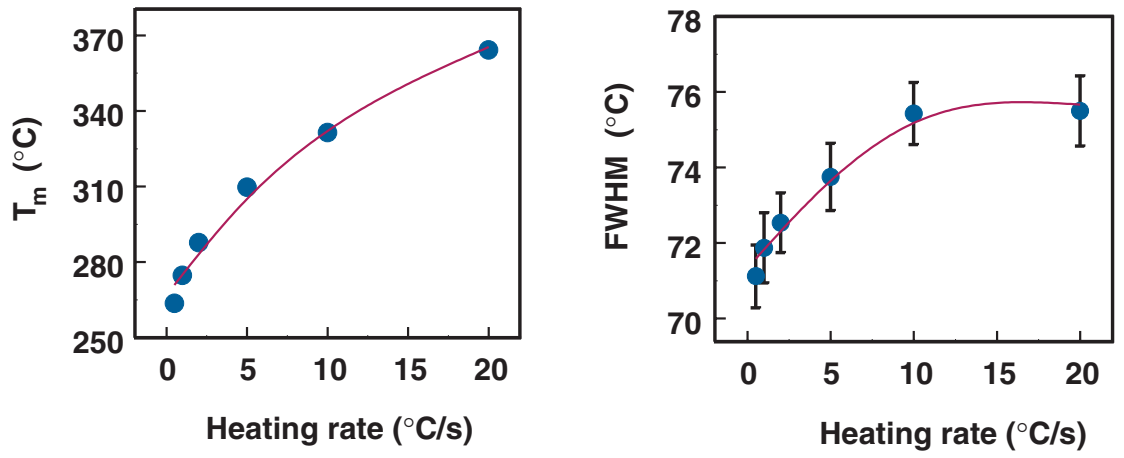

Fig. 3 (online colour at: www.pss-a.com) Left-hand side: The position of the peak maximum temperature $T_{\mathrm{m}}$ of the main glow-peak of Durango apatite as a function of the heating rate. Right-hand side: The FWHM of the main glow-peak as a function of the heating rate.

be very similar to that of the peak maximum temperature $T_{\mathrm{m}}$, which is shown in the left-hand side of Fig. 3.

The TL signal is the remnant TL i.e. the TL remaining after the storage of the sample at room temperature for various time intervals from the end of irradiation. The remnant TL is defined by the parameter $r$, which is the ratio of the TL at time $t$ over to the initial TL at time $t_{0}$. In the case of the tunnelling model for anomalous fading phenomenon, the remnant TL ratio $r$ is described by an equation of the form $[3,4,18]$

$$
r=A-K \cdot \ln \left(\frac{t}{t_{0}}\right),
$$

where $A$ and $K$ represent constants characterizing the anomalous fading of the material and $A$ is usually taken to be equal to 1 . However, for fitting needs, the parameter $A$ can be considered as a free parameter varying around 1 .

The fading rate in terms of percentage per decade, $g$, [19] is related to $K[2,20]$ by

$$
g=230.2 \cdot K .
$$

Figure 4 shows the apparent anomalous fading of TL for various heating rates. As can be seen in Fig. 4 the behavior changes substantially from the low heating rate of $0.5^{\circ} \mathrm{C} / \mathrm{s}$ shown in curve (a), to the highest heating rate of $20^{\circ} \mathrm{C} / \mathrm{s}$ shown in curve (f). This is clearly shown in Fig. 5, which shows how the remnant TL factor, $r$, as a function of time, varies with the heating rate for various storage times. The

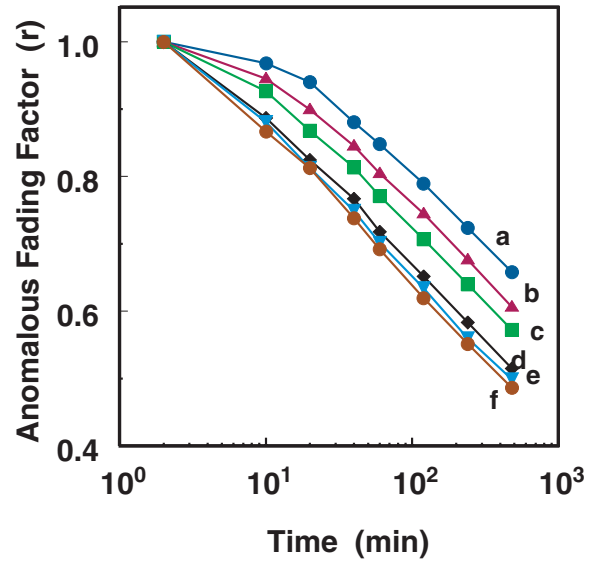

Fig. 4 (online colour at: www.pss-a.com) Apparent anomalous fading factor $r$ of the integral of the main glow-peak as a function of the storage time elapsed from the end of irradiation, normalized over the zero storage time for various heating rates (a) 0.5 , (b) 1 , (c) 2 , (d) 5 , (e) 10 and (f) $20^{\circ} \mathrm{C} / \mathrm{s}$. 


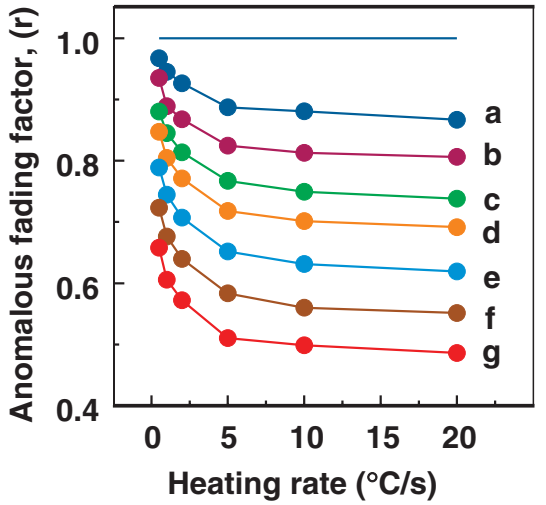

Fig. 5 (online colour at: www.pss-a.com) Apparent anomalous fading factor $r$ of the integral of the main glow-peak as a function of the heating rate for various storage times. (a) $10 \mathrm{~min}$, (b) $20 \mathrm{~min}$ (c) 40 min, (d) 60 min, (e) $120 \mathrm{~min}$ (f) $240 \mathrm{~min}$ and (g) 480 min. The solid line at unity corresponds to zero storage time.

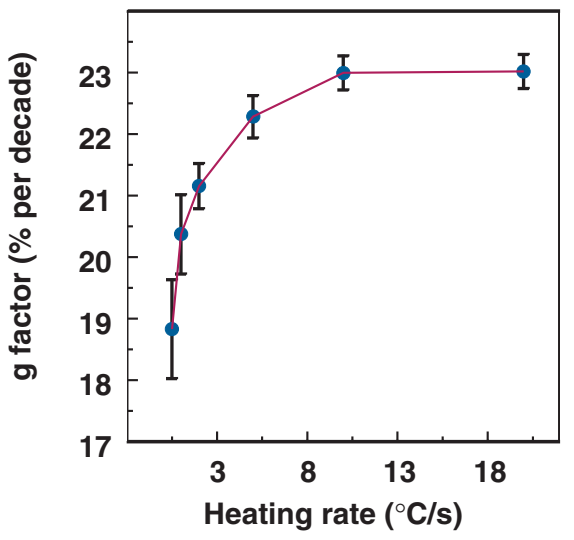

Fig. 6 (online colour at: www.pss-a.com) $g$ factor in $\%$ per decade as a function of the heating rate.

tunnelling model for AF predicts that the behavior of the remnant TL, $r$, as a function of $\ln (t)$ is linear (Eq. (1)). For the lower heating rates $\left(0.5-1{ }^{\circ} \mathrm{C} / \mathrm{s}\right)$ there is some deviation from linearity as can be seen in Fig. 4. As the heating rate increases, the behavior becomes clearly linear in full agreement with the predictions of the tunnelling model. By fitting the experimental data to Eq. (1), the value of $K$ can be evaluated from the slope and the values of the $g$ factor can be estimated from Eq. (2). The behavior of the $g$ factor as a function of the heating rate is shown in Fig. 6. As can be seen for Fig. 6, the AF factor $g$ increases as the heating rate used in recording the TL glow-curve increases.

\section{Discussion}

\subsection{Dependence of the TL response on the heating rate}

The predictions of the TL kinetics theory concerning the TL as a function of the heating rate are as follows:

As the heating rate increases:

1. the peak maximum temperature, $T_{\mathrm{m}}$, shifts to higher temperatures

2. the Full Width at Half Maximum (FWHM) increases

3. the integral of the glow-peak is always the same (theoretically equal to the number of trapped electrons $n_{0}$ )

4. the maximum TL intensity, $I_{\mathrm{m}}$, decreases slightly, since the glow-peak integral remains stable and the FWHM increases.

From the above predictions, only the behavior of $T_{\mathrm{m}}$ was verified (left-hand side of Fig. 3 ) in the present work. The variation of the FWHM is unexpectedly small (right-hand side of Fig. 3). The integral of the glow-peak increases as the heating rate increases (Fig. 3), whereas the $I_{\mathrm{m}}$ follows the behavior of the integral due to the very small variation of the FWHM.

It must be noted that it is not possible to present the exact numerical variations of these quantities with the heating rate according to standard kinetic models, due to the fact that these variations depend on the exact parameters (energy, frequency factors etc.) used in the models. 
The decrease of the TL response as a function of the heating rate is a very well known and widely studied phenomenon attributed to the thermal quenching effect $[10,11]$. The effect is very large in the case of the sensitive TL dosemeter $\mathrm{Al}_{2} \mathrm{O}_{3}: \mathrm{C}[10,12]$. Akselrod et al. [12] proved convincingly that the thermal quenching effect is responsible for the decrease of the TL response as a function of the heating rate for this material, and evaluated accurately the thermal quenching activation energy and the corresponding frequency factor. On the other hand, to the best of our knowledge, there are currently no satisfactory explanations for the increase of the TL as a function of the heating rate observed in this study. A possible explanation is that the increase of the TL as a function of the heating rate is due to an increase of the luminescence efficiency $\eta$, which in phenomenological TL kinetics models is taken equal to unity for the sake of simplicity.

According to Bos [21] the intrinsic energy efficiency $\eta_{\mathrm{i}}$ of a TL material is defined as the ratio of the energy emitted as light during heating, to the energy absorbed during irradiation. The final expression for the $\eta_{\mathrm{i}}$ given by Bos [21] is

$$
\eta_{\mathrm{i}}=\frac{h v}{\beta E_{\mathrm{g}}} n_{\mathrm{tr}} p S Q \eta_{\mathrm{esc}}
$$

where $h v$ the average energy of the emitted TL photon, $E_{\mathrm{g}}$ the forbidden bad gap width, $\beta$ has a value depending upon the type of material [22], $n_{\mathrm{tr}}$ is the fraction of charge carriers that are captured in traps and which can be thermally stimulated, $p$ the probability of charged carriers escaping from the traps and transporting to luminescence centers with an efficiency $S$, exciting the luminescence centers that deexcite under emission of photons with a quantum efficiency $Q$. Finally, $\eta_{\text {esc }}$ is the fraction of produced TL photons that escape from the sample without being absorbed by it.

For the experimental conditions of the present work the quantities $\beta$ and $E_{\mathrm{g}}$ concern the material itself and cannot depend on the heating rate. The quantity $n_{\mathrm{tr}}$ concerns the irradiation stage of the material and must be taken as a constant since the pre-irradiation treatment and the irradiation test dose are always the same. The parameter $\eta_{\text {esc }}$ is involved during the heating stage but is also characteristic of the sample, so it must also be considered a constant. Therefore, all of these parameters can be represented by a constant $C$ and Eq. (3) becomes

$$
\eta_{\mathrm{i}}=C p S Q \text {. }
$$

Under these circumstances only the quantities $p, S$ and $Q$ can vary with the heating rate. These constants make-up the luminescence efficiency $\eta_{\mathrm{TL}}[21]$, i.e.,

$$
\eta_{\mathrm{TL}}=p S Q
$$

Since all the trapped electrons are freed during readout, the probability $p=1$. The quantum efficiency $Q$ must be taken as a constant, because when the heating rate increases the value of $Q$ is expected to decrease, as in the case of the thermal quenching effect, and not to increase. Therefore, the effect of TL increase as a function of heating rate is most probably due to the variation of the rate $S$ by which the freed electrons are transferred to the luminescence centers.

Since the whole glow-peak shifts to higher temperatures as the heating rate increases, it is concluded that the value of $S$ should be temperature dependent. This can be seen if instead of plotting the TL as a function of heating rate as in (Fig. 2), one plots the TL as a function of the $T_{\mathrm{m}}$ for the corresponding heating rate. This technique was used for $\mathrm{Al}_{2} \mathrm{O}_{3}: \mathrm{C}[10,12]$ and for quartz [23], in order to evaluate the activation energy for thermal quenching.

These types of plots are shown on the left-hand side of Fig. 7, where one can see that the TL integral is a linear function of the maximum peak temperature (with a regression coefficient $>0.995$ for seven experimental points). The slope of each line, shown in the right-hand side of Fig. 7, gives the rate by which the freed electrons are transferred to the luminescence centers. The interesting result here is that the slope decreases as the storage time increases. Since anomalous fading takes place during the storage 

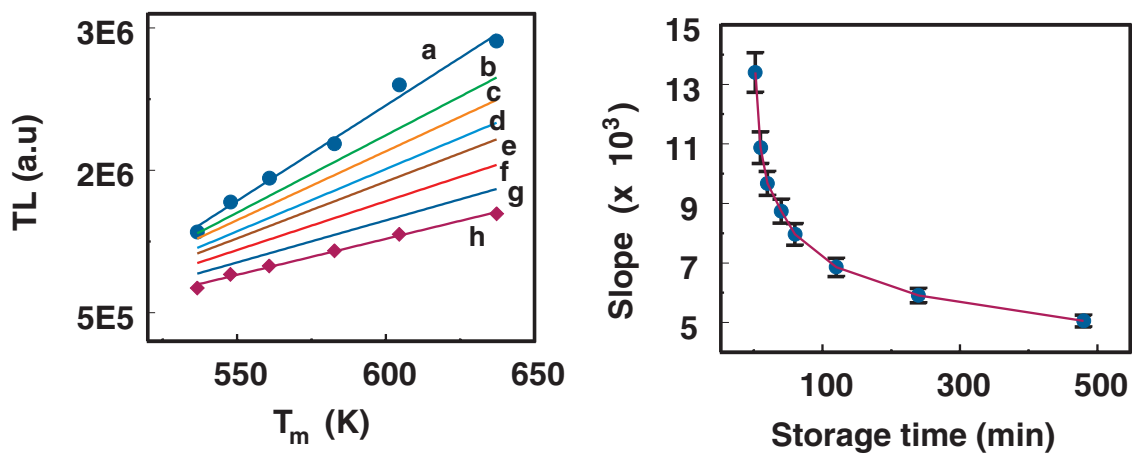

Fig. 7 (online colour at: www.pss-a.com) Left-hand side: The TL response as a function of the peak maximum temperature corresponding to the heating rate used, for various storage times. Line (a) corresponds to zero storage time, and the intermediate lines $(b-h)$, for which the experimental points are not drown, correspond to storage times 10, 20, 40, 60, 120, 240 and $480 \mathrm{~min}$, respectively. Right-hand side: The slope of the straight lines of the left-hand side figure as a function of the storage time.

time, the concentration of trapped electrons $n_{0}$, which are available during the readout decreases with the storage time. In the simplest model [1] (p. 26) the electrons are freed from their traps into the conduction band, whose concentration is denoted by $n_{\mathrm{c}}$ and are transferred to the luminescence centers with a rate given by $n_{\mathrm{c}} \cdot A_{\mathrm{m}}$, where $A_{\mathrm{m}}$ is called the recombination probability in the phenomenological TL kinetics models [1]. The same conclusion will be reached if re-trapping is also taken into account. The interesting point is that the recombination probability, $A_{\mathrm{m}}$, is considered to be temperature dependent [1]. This is experimentally verified in the present work, thus explaining the increase of the TL response as a function of the heating rate.

\subsection{Apparent anomalous fading factor versus heating rate}

This result is shown in Fig. 2. The conclusion from this figure is that the apparent anomalous fading factor is found to be larger when high readout heating rates are used. The remnant TL factor, $r$, is obtained by dividing the TL after various storage times by the TL after zero storage time. However, as it is seen from Fig. 2 the dependence of the TL on the heating rate is greater for the case of zero storage time. This means that the respective TL responses for longer storage times are normalized over the strongest $\mathrm{TL}$ response at zero storage time. Therefore, in order to explain the results of Fig. 5, one must explain why the dependence of TL versus heating rate becomes weaker as the storage time increases.

The real anomalous fading, obviously, takes place during the storage time. Therefore, the anomalous fading factor (remnant TL, $r$ ), should be always the same, independent of the readout conditions. The anomalous fading during storage alters (decreases) the concentration of trapped electrons $n_{0}$. This means that for each storage time a different concentration $n_{0 i}$, with $i$ the storage time, is available during the TL readout.

However, during TL readout the $n_{0 \mathrm{i}}$ electrons are released from the TL traps and contribute to the respective concentration $n_{\mathrm{ci}}$ of electrons in the conduction band. As was discussed above, the product of the concentration $n_{\mathrm{ci}}$ and the recombination probability $A_{\mathrm{m}}$ gives the rate at which the freed electrons are transferred to the luminescence centers. The latter is the slope of the TL versus $T_{\mathrm{m}}$ lines shown on the left-hand side of Fig. 7. Since the number of $n_{0 \mathrm{i}}$ decreases as a function of storage time, the slope will decrease accordingly, as shown in the right-hand side of Fig. 7. In conclusion, the variation of the heating rate dependence of TL due to storage time is due to the combined effects of a temperature dependence of the recombination probability $A_{\mathrm{m}}$ and a decrease in the concentration of trapped electrons due to anomalous fading during storage. 


\section{Conclusions}

1. The TL response of the Durango apatite was found to increase as the heating rate increases, instead of the constancy expected from the phenomenological kinetic models.

2. The TL response of Durango apatite depends linearly on the peak maximum temperature obtained at each heating rate. Based on this observation, the results of this study show that the luminescence efficiency in Durango apatite must increase linearly as a function of temperature.

3. It is suggested that the linear increase of the luminescence efficiency as a function of temperature is responsible for the increase of the TL response as a function of the heating rate.

4. The apparent AF factor $r$ of Durango apatite evaluated by monitoring the remnant TL, was found to decrease as the heating rate increases.

5. This last experimental result was attributed to a combination of two effects. (i) to the decrease of the number of trapped electrons in the trap responsible for the TL as the storage time increases, and (ii) to the decrease of the slope of the linear dependence of the luminescence efficiency during readout with the storage time.

\section{References}

[1] R. Chen and S. W. S. McKeever, Theory of Thermoluminescence and Related Phenomena (World Scientific, 1997), p. 454.

[2] L. Bøtter-Jensen, S. W. S. McKeever, and A. Wintle, Optically Stimulated Luminescence Dosimetry (Elsevier, 2003).

[3] R. Visocekas, T. Ceva, C. Marti, F. Lafaucheux, and M. C. Robert, phys. stat. sol. (a) 35, 315 (1976).

[4] R. Visocekas, Nucl. Tracks 10, 521 (1985).

[5] R. H. Templer, Radiat. Prot. Dosim. 17, 493 (1986).

[6] D. C. W. Sanderson, Nucl. Tracks Radiat. Meas. 14, 155 (1988).

[7] R. Chen and A. Hag-Yahya, Radiat. Meas. 27, 205 (1997).

[8] G. Kitis, P. Bousbouras, C. Antypas, and S. Charalambous, Nucl. Tracks Radiat. Meas. 18, 61 (1991).

[9] G. Polymeris, N. Tsirliganis, Z. Loukou, and G. Kitis, phys. stat. sol. (a) 203, 578 (2006).

[10] G. Kitis, J. G. Papadopoulos, S. Charalambous, and J. W. N. Tuyn, Radiat. Prot. Dosim. 55, 183 (1994).

[11] S. G. Gorbics, A. E. Nash, and F. H. Attix, in: Proc. Second International Conference on Luminescence Dosimetry, Gatliburg, TN, USA, 1968, edited by J. A. Auxier, K. Becker, and E. M. Robinson, p. 587.

[12] M. Akselrod, N. Agersnap Larsen, V. Whitley, and S. W. S. McKeever, J. Phys. D: Appl. Phys. 84, 3364 (1988).

[13] E. F. Mische and S. W. S. McKeever, Radiat. Prot. Dosim. 29, 159 (1989).

[14] R. Chen, X. H. Yang, and S. W. S. McKeever, J. Phys. D: Appl. Phys. 21, 1452 (1988).

[15] D. Zimmerman, Archaeometry 13, 29 (1971).

[16] D. S. Betts and P. D. Townsend, J. Phys. D: Appl. Phys. 26, 849 (1993).

[17] G. Kitis and J. W. N. Tuyn, J. Phys. D: Appl. Phys. 31, 2065 (1998).

[18] M. Auclair, M. Lamothe, and S. Huot, Radiat. Meas. 37, 487 (2003).

[19] M. J. Aitken, Thermoluminescence dating (Academic Press, 1985), Appendix F.

[20] M. Lamothe, M. Auclair, C. Hamzaoui, and S. Huot, Radiat. Meas. 37, 493 (2003).

[21] A. J. J. Bos, Radiat. Meas. 33, 737 (2001).

[22] P. A. Rodnyi, Radiat. Meas. 29, 235 (1998).

[23] G. Kitis, V. Pagonis, and C. Drupieski, phys. stat. sol. (a) 198, 312 (2003). 